University of Nebraska - Lincoln

DigitalCommons@University of Nebraska - Lincoln

\title{
Transcriptional Regulation of the Murine Elf3 Gene in Embryonal Carcinoma Cells and Their Differentiated Counterparts: Requirement for a Novel Upstream Regulatory Region
}

Jingwen Hou

Eppley Institute for Research in Cancer and Allied Diseases at the University of Nebraska Medical Center, USA

Phillip J. Wilder

Eppley Institute for Research in Cancer and Allied Diseases at the University of Nebraska Medical Center, USA

Cory T. Bernadt

Eppley Institute for Research in Cancer and Allied Diseases at the University of Nebraska Medical Center, USA

Brian Boer

Eppley Institute for Research in Cancer and Allied Diseases at the University of Nebraska Medical Center, USA

Richard M. Neve

fife Science Divisjan Layrence Berkeley National Laboratory, 1 Cyclotron Road Berkeley, CA 94720, USA

aplato

Hou, Jingwen; Wilder, Phillip J.; Bernadt, Cory T.; Boer, Brian; Neve, Richard M.; and Rizzino, Angie, "Transcriptional Regulation of the Murine Elf3 Gene in Embryonal Carcinoma Cells and Their Differentiated Counterparts: Requirement for a Novel Upstream Regulatory Region" (2004). Public Health Resources. 72.

https://digitalcommons.unl.edu/publichealthresources/72

This Article is brought to you for free and open access by the Public Health Resources at DigitalCommons@University of Nebraska - Lincoln. It has been accepted for inclusion in Public Health Resources by an authorized administrator of DigitalCommons@University of Nebraska - Lincoln. 


\section{Authors}

Jingwen Hou, Phillip J. Wilder, Cory T. Bernadt, Brian Boer, Richard M. Neve, and Angie Rizzino 


\title{
Transcriptional regulation of the murine Elf3 gene in embryonal carcinoma cells and their differentiated counterparts: requirement for a novel upstream regulatory region
}

\author{
Jingwen Hou ${ }^{\mathrm{a}}$, Phillip J. Wilder ${ }^{\mathrm{a}}$, Cory T. Bernadt ${ }^{\mathrm{a}, \mathrm{b}}$, Brian Boer ${ }^{\mathrm{a}, \mathrm{b}}$, \\ Richard M. Neve ${ }^{c}$, Angie Rizzino ${ }^{a, b, *}$ \\ appley Institute for Research in Cancer and Allied Diseases at the University of Nebraska Medical Center, USA \\ ${ }^{\mathrm{b}}$ Department of Pathology and Microbiology at the University of Nebraska Medical Center, USA \\ ${ }^{\mathrm{c}}$ Life Science Division, Lawrence Berkeley National Laboratory, 1 Cyclotron Road, Berkeley, CA 94720, USA
}

Received 9 April 2004; received in revised form 20 May 2004; accepted 1 June 2004

Available online 23 July 2004

Received by A.J. van Wijnen

\begin{abstract}
The transcription factor Elf3, which is one of over 25 Ets family members, is expressed in a wide variety of carcinomas and has been shown to promote the transcription of many genes implicated in cancer. To understand how the Elf3 gene is regulated at the transcriptional level, we probed its $5^{\prime}$-flanking region, and we report here the identification of both proximal and distal regions that regulate murine Elf3 promoter activity. In addition to mapping the transcription start site of the Elf3 gene, the work described in this study identifies four cisregulatory elements in the proximal promoter region of the gene. These include a cis-regulatory element previously designated ESE, a $\kappa \mathrm{B}$ site, a POU motif, and a CCAAT box. In addition, we demonstrate that a novel 94 bp region $2 \mathrm{~kb}$ upstream of the transcription start site significantly elevates Elf3 promoter activity in F9-differentiated cells, but not in the parental F9 embryonal carcinoma (EC) cells. This region appears to be largely responsible for the increase in Elf3 promoter activity that accompanies the differentiation of embryonal carcinoma cells.
\end{abstract}

Keywords: Ets proteins; Differentiation; Gene regulation; Distal enhancer; ESX; ESE1; ERT; Jen

\section{Introduction}

Transcription factors belonging to the Ets family play critical roles in normal growth and development, as well as human malignancy (Dittmer and Nordheim, 1998). Since the discovery of the first Ets family member, over 50 Ets genes have been identified, and at least 25 are expressed in mammals (Laudet et al., 1999). One Ets family member, Elf3 (also known as ESX, ESE-1, ERT,

Abbreviations: EC, embryonal carcinoma; RA, retinoic acid; T $\beta R-I I$, type II TGF- $\beta$-receptor; 5'-RACE, rapid amplification of cDNA ends; EMSA, electrophoretic mobility shift assay.

* Corresponding author. Eppley Institute for Research in Cancer and Allied Diseases, 986805 Nebraska Medical Center, Omaha, NE 681986805, USA. Tel.: +1-402-559-6338; fax: +1-402-559-4651.

E-mail address: arizzino@unmc.edu (A. Rizzino). and JEN), is expressed ubiquitously by epithelial cells (Chang et al., 1997; Oettgen et al., 1997; Tymms et al., 1997), but it also can be induced in other cell types under pathological conditions (Grall et al., 2003). The roles of Elf3 have been examined recently in both cell culture and animal models. Inactivation of both alleles of Elf3 by gene targeting leads to fetal lethality (Ng et al., 2002). Approximately $30 \%$ of the null fetuses die around embryonic day 11.5. The $70 \%$ that survive to birth exhibit severe alterations in the cellular architecture of the small intestine, including poor villus formation and defective terminal differentiation of the mucus-secreting goblet cells and the absorptive enterocytes. Moreover, enterocytes from Elf3 null animals express significantly reduced levels of type II TGF- $\beta$-receptor (T $\beta R$-II) at both the RNA and the protein level. Similarly, reduction of Elf3 in a colon cancer cell line by use of an Elf3 antisense retroviral construct 
also reduced $T \beta R$-II expression (Lee et al., 2003). Other studies have linked Elf3 directly to $T \beta R$-II transcription in epithelial cells by its binding to two essential ets-sites in the TRR-II promoter (Choi et al., 1998; Kim et al., 2002; Kopp et al., 2004). In addition to the $T \beta R-I I$ gene, Elf3 has been implicated in the regulation of multiple genes, including endo A, SPRR2A, SPRRIB, and nitric-oxide synthase (Oettgen et al., 1997; Rudders et al., 2001; Reddy et al., 2003).

Elf3 also appears to play important roles in cancer, and it is aberrantly expressed in cancers of the lung and breast (Chang et al., 1997). In the lung, elevated Elf3 expression has been implicated in the expression of the SPRRIB gene, a marker for early metaplastic alterations in bronchial epithelium (Reddy et al., 2003). In breast cancer, the $1 \mathrm{q} 32$ chromosomal region containing Elf3 (Chang et al., 1997) is amplified in $50 \%$ of early tumors (Isola et al., 1995). However, in the case of Hs578t breast cancer cells, Elf3 is expressed at very low levels. Elevating the expression of Elf3 in Hs578t cells dramatically elevates the expression of $T \beta R-I I$ and decreases the tumorigenicity of these cells (Chang et al., 2000).

Given the important roles of Elf3 in normal cellular physiology and cancer, it is important to determine how transcription of the Elf3 gene is regulated. Sequence analyses of the Elf 3 gene have identified several potential transcription factor binding sites in its promoter region, including ets, POU, USF, and $\kappa \mathrm{B}$ sites, as well as GC, CCAAT, and TATA boxes (Neve et al., 1998; Oettgen et al., 1999). Thus far, only the $\kappa B$ site and a novel site, termed an ESE element, in the human ELF3 gene have been shown to be functional (Rudders et al., 2001; Park et al., 2001). Importantly, other regions of the Elf3 gene have not been examined for their effects on its transcription.

The aim of this study was to identify and characterize cisacting regions of the murine Elf3 gene that control its transcription in early embryonic tumor cells. This work was conducted using murine embryonal carcinoma (EC) cells and their retinoic acid (RA) induced differentiated counterparts, because the steady-state levels of Elf3 mRNA increase at least sixfold when F9 EC cells differentiate (Kim et al., 2002). Currently, it is unknown whether increases in Elf3 expression influence the differentiation of EC cells, but it is believed to play an important role in the increase in transcription of the TRR-II gene after EC cells differentiate (Kim et al., 2002).

In this study, we have identified two regions of the Elf3 gene that influence the activity of its promoter. Initially, we mapped the transcription start site of the gene in F9differentiated cells and tested the function of six potential cis-regulatory elements in the proximal promoter region of the murine Elf3 gene. Importantly, we also demonstrate that differentiation substantially increases the activity of the Elf3 promoter and that this increase in promoter activity is due in large measure to a novel regulatory region located approximately $2 \mathrm{~kb}$ upstream of the transcription start site.

\section{Materials and methods}

\subsection{Cell culture, transfection and luciferase assay}

F9 EC cells were cultured on gelatinized dishes in Dulbecco's modified Eagle's medium (Invitrogen, Carlsbad, CA) supplemented with $10 \%$ fetal bovine serum (HyClone, Logan, UT) and antibiotics (Kim et al., 2002; Nowling et al., 2003). Unless otherwise stated, all chemicals were purchased from Sigma (St. Louis, MO). F9 EC cells were transfected on the day after being seeded at 20,000 cells/well in 24-well plates. For transfection of F9-differentiated cells, F9 EC cells were seeded at 4000 cells/well in 24-well plates and treated with $5 \mu \mathrm{M}$ RA to induce differentiation. The differentiated cells were transfected 3 days later. Transfections were performed with Lipofectamine and the Plus transfection reagent (Invitrogen) according to the manufacturer's instructions using $400 \mathrm{ng}$ of Elf3 promoter-luciferase plasmids and $200 \mathrm{ng}$ of pRL-TK internal control vector (Promega, Madison, WI). Cells were harvested $24 \mathrm{~h}$ after transfection, luciferase activity was measured using a Dualluciferase reporter assay kit (Promega) with a Luminoskan RS plate-reading luminometer (MTX Lab Systems, Vienna, VA), and activities were normalized to the activity of an internal control vector, pRL-TK. Values are presented as the mean \pm standard error of the mean (S.E.M.) of duplicate or triplicate samples.

\subsection{Sequencing}

The LI-COR and ABI Sequencers at the Genomics Core Research Facility at the University of Nebraska at Lincoln were used along with primer walking to sequence the $5^{\prime}$ flanking region of the mouse Elf3 gene. Both strands were sequenced. The ABI sequencer was also used to verify critical sequences in various plasmids.

\subsection{Mapping the transcription start site by rapid amplification of $c D N A$ ends $\left(5^{\prime}-R A C E\right)$}

The transcription start site of the murine Elf3 gene was determined using 5'-RACE. Two internal primers, $5^{\prime}$ TCTCCCAGAGGTCCAAAGACTAGC-3' (nucleotide position 378 to 401) and 5'-AGCTCCTCCAGCGCACAGCTGCAGAGGGTGGCTCC-3' (nucleotide position 340 to 374), were designed based on the Elf3 cDNA sequence (GenBank NM-007921). These two primers were used in conjunction with the RLM 5'-RACE adapter primer (Ambion, Austin, TX) to capture the $5^{\prime}$ end of the Elf3 mRNA. The resulting fragments were cloned into the pSP72 vector (Promega). The inserts of 17 recombinant clones were sequenced. 


\subsection{Generation of promoter/reporter gene constructs}

The Elf3 promoter region -338 to +122 was amplified by PCR from genomic DNA of F9-EC cells. Restriction sites NheI and BglII were incorporated at the ends of the PCR primers to allow insertion into the pGL3-luciferase reporter vector (Promega). PCR primers were designed based on the promoter region sequence from Neve et al. (1998) 5' GTAGCTAGCGCCAGGCCCCCAGGAAGA-3' (sense) and the cDNA sequence of Tymms et al. (1997) 5'-GTAAGATCTGGTGTGGCAGGCGGGTGA-3' (antisense). To mutate potential cis-regulatory elements within this sequence, site-directed mutagenesis of the Elf3 promoter Luciferase construct was performed using the "quick change method", as described previously (Kim et al., 2002; Nowling et al., 2003). The following primers were used along with their reverse complements: 5' CAGATTCTGACAATCATTAAAACTCGAGGCCTCTGATTTCCAG-3' for ESE mut $^{\prime}$ 5'-CAGGAAATCCCCAGCATCTCGAGAGCCACCAGCTCAGGTTAC-3' for $\mathrm{CCAAT}_{\text {mut }}$; 5'-AAGTGGCACGGAATATGAATTTCACCTGGGACAGGGAGCCCAG-3' for POU Put $^{\prime}$ and 5'-GGGAGCCCAGTCTGAAGGCCAGTTAATTAACAGCATCCAATGAGC- $3^{\prime}$ for $\kappa B_{\text {mut }}$. The bolded nucleotides are the mutations introduced into the sequence. All promoter sequences were verified by DNA sequencing.

The region of the mouse Elf3 gene from - 2898 to +703 was inserted into the pGL3-Basic vector between the MluI and HindIII sites to generate the promoter/ reporter construct, $m E l f 3-2898 /+703$. Additional promoter/reporter gene constructs lacking portions of this sequence were constructed by digestion with pairs of restriction enzymes, blunting with klenow, and religation. Thus, $m E l f 3-2437 /+703$ was generated using MluI and KpnI, mElf3 - 2347/+703 using MluI and BglII, $m E l f 3-2012 /+703$ using MluI and EcoRI, and

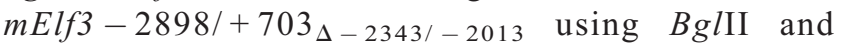
EcoRI.

To generate $m E l f 3-338 /+703$, the promoter region upstream of +32 was removed from $m$ Elf $3-2898 /+703$ and the fragment $-338 /+32$ was obtained from $m E l f 3-338 /+122$ by digestion with $K p n I$ (at an upstream polylinker site) and $M s c I$. The $-338 /+32$ fragment was then inserted into what was left of $m$ Elf3 $-2898 /+703$. Additional promoter/reporter constructs were made by inserting various sequences between the KpnI and NheI restriction sites upstream of the promoter in $m E l f 3-338$ / +703 . Three constructs made in this manner using sequences generated by PCR from mElf3 - 2898/-703 were $m$ Elf3 - 2347/ + 703 $3_{\Delta-2012 /-339, \quad m E l f 3-2347 /}$ $+703_{\Delta-2211 /-339}$, and $m E l f 3-2236 /+703_{\Delta-2142 /-339}$. Another series of constructs made in this manner included various mutations of the Elf3 sequence from -2236 to -2143 . These mutations were substitutions of 'CCCGGG' in each of 15 positions in the sequence (or ' $\mathrm{CGG}$ ' in place of the first three bases). In these cases, the sequences to be inserted were obtained by synthesis of smaller overlapping oligonucleotides, which were extended using taq polymerase. All promoter sequences were verified by DNA sequencing.

The promoter/reporter construct, $m E$ lf $3-2347 /$ $+703_{\Delta-2012 /-339}$, was used in turn to generate two additional constructs. $m E l f 3-2347 /+703_{\Delta}-2012 /-339$ was digested with pairs of restriction enzymes, KpnI and EcoRI or EcoRI and NheI, to produce mElf3-2093/ $+703_{\Delta-2012 /-339}$ and $m E l f 3-2347 /+703_{\Delta-2089 /-339}$, respectively, after blunting with klenow and religation. All promoter sequences were verified by DNA sequencing.

\section{Results}

\subsection{Sequence of the murine Elf3 $5^{\prime}$-flanking region}

The primary objective of this study was to identify regions of the Elf3 gene required for its transcription. To initiate this study, the murine Elf3 gene from -2898 to +703 was sequenced. This sequence has been assigned the GenBank accession number AY456682. To facilitate comparison with previous studies, we have designated the nucleotide positions in this report according to the numerical system of Neve et al. (1998), who presented an alignment of the human ELF3 and murine Elf3 promoters between -347 and +50 of the murine sequence.

\subsection{Mapping the transcription start site of the murine Elf3} gene

The only published report concerning the transcription start site of the murine Elf3 gene places it $50 \mathrm{bp}$ downstream from a consensus TATA box (Tymms et al., 1997). However, functional TATA boxes typically direct transcriptional initiation to locations $30 \pm 2 \mathrm{bp}$ downstream (Bucher, 1990). Therefore, we mapped the location of the transcription start site of the murine Elf3 gene in F9-differentiated cells. For this purpose, 5'RACE was used. Seventeen clones were isolated and sequenced, and a dominant transcriptional initiation site (16 out of 17 clones) was identified at nucleotide +32 (Fig. 1). This site is $30 \mathrm{bp}$ downstream of the putative TATA box located at +2 . The sequence of the 17 th clone in our study corresponds to a start site at nucleotide +33 , and it could have resulted from premature termination during the polymerization step. Thus, it appears that one major start site is employed in F9differentiated cells. Interestingly, sequence analysis of our 17 clones identified both splice variants of Elf3 mRNA, Elf3a and Elf3b, observed previously in the adult lung of mice (Tymms et al., 1997). Thus, both Elf3a and Elf3b, which differ by 20 amino acids, may be expressed in F9differentiated cells. 


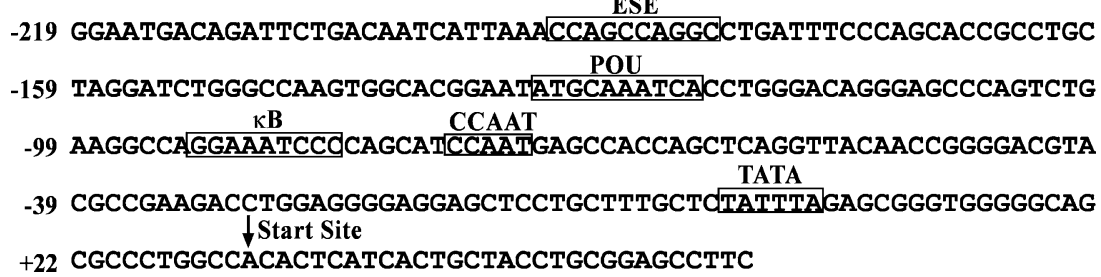

Fig. 1. Nucleotide sequence of the region containing the murine Elf3 transcription start site. The transcription start site of murine Elf3 in F9-differentiated cells was determined by $5^{\prime}$-RACE as described in Materials and methods. The major transcription start site is indicated by an arrow. Other sequences of interest are boxed, including potential ESE, POU, and $\kappa \mathrm{B}$ elements and CCAAT and TATA boxes.

\subsection{The proximal promoter region of Elf3 contains at least four cis-regulatory elements}

To identify essential cis-regulatory elements in the murine Elf3 gene, we inserted the region between -338 and +122 of the Elf3 gene into the promoterless luciferase reporter gene construct, pGL3 Basic, to create $m E l f 3-338 /+122$. This construct exhibited 100-fold higher luciferase activity than the parent pGL3 Basic vector (data not shown). Previous analysis of this region (Neve et al., 1998) identified several potential cis-regulatory elements, including a GC box, a USF site, a POU motif, a $\kappa \mathrm{B}$ site and a CCAAT box. Of these, only the $\mathrm{\kappa B}$ site has been shown to be functional in the human ELF3 gene (Rudders et al., 2001). In addition, a novel cisregulatory element referred to as ESE was identified recently in the human ELF3 gene and shown to act as a positive cisregulatory element in a human gastric cancer cell line (Park et al., 2001). To determine whether any of these potential regulatory sequences are functional in the murine Elf 3 gene, each of these sites were disrupted individually in the Elf3 promoter/reporter gene construct $m E l f 3-338 /+122$. Disruption of the ESE element and the $\mathrm{kB}$ site reduced promoter activity by $48 \%$ and $43 \%$, respectively (Fig. 2). Larger reductions in promoter activity were observed when the POU motif and CCAAT box were mutated, $65 \%$ and $71 \%$, respectively. However, disruption of the two GC boxes or the
USF site did not reduce Elf3 promoter activity (data not shown). Thus, the proximal promoter region of the Elf3 gene contains at least four $\mathrm{cis}$-regulatory elements that influence its activity, a CCAAT box, a $\kappa \mathrm{B}$ site, a POU motif and ESE.

\subsection{Differentiation of F9 EC cells elevates Elf3 promoter activity}

Previous studies demonstrated that differentiation of F9 EC cells leads to significant increases in the steady-state levels of Elf3 mRNA (Kim et al., 2002). Therefore, we examined whether the activity of the Elf3 promoter increases when EC cells differentiate. Initially, this was examined by transiently transfecting F9 EC cells with $m E L F 3-338 /+122$. Five hours after transfecting the cells, the medium was changed and RA was added to one set of F9 EC cells to induce differentiation. In comparison to the untreated F9 EC cells, Elf3 promoter activity increased approximately twofold after a 3-day exposure to RA (Fig. $3 \mathrm{~A})$. This was significantly lower than the sixfold increase in Elf3 mRNA observed previously when F9 EC cells differentiate (Kim et al., 2002). This raised the possibility that other regions of the Elf3 gene are required for full promoter activity in F9-differentiated cells. To examine this possibility, we generated a murine Elf3 promoter/reporter gene construct that contains a larger region of the Elf3 gene.

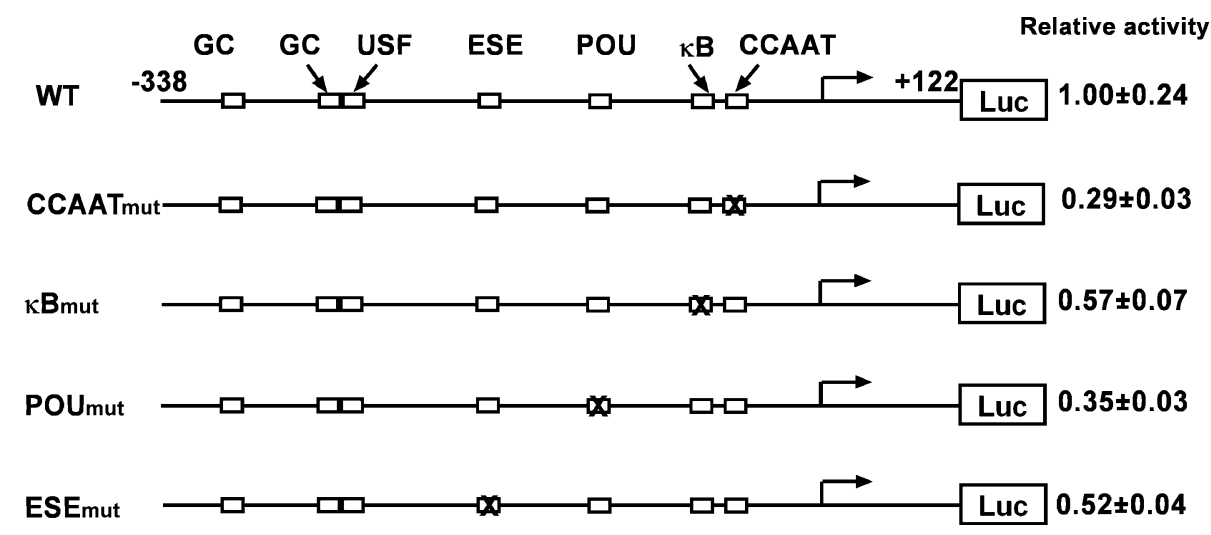

Fig. 2. Murine Elf3 promoter/reporter activity in F9-differentiated cells. Promoter/reporter constructs with potential cis-regulatory sites mutated (as indicated with an " $\mathrm{X}$ ") were transiently transfected into F9-differentiated cells and assayed for luciferase activity after $48 \mathrm{~h}$ as described in Materials and methods. Results were normalized with an internal control and expressed relative to the activity of the unmutated, wild-type, construct $m E l f 3-338 /+122$. Values shown are averages \pm S.E.M. for duplicate samples in a representative experiment. This experiment was performed three times with similar results. 
Specifically, we inserted the region -2898 to +703 into the promoterless vector pGL3 Basic to generate mElf3 $2898 /+703$. When this construct was transfected into F9 EC cells, we observed a fivefold increase in promoter activity for F9 cells exposed to RA for 3 days (Fig. 3A). To confirm and extend these findings, we directly compared the activity of $m E l f 3-2898 /+703$ to that of $m E L F 3-338 /+122$. There was no significant difference between the activity of the larger and the smaller Elf3 promoter/reporter gene constructs when they were transiently transfected into F9 EC cells (Fig. 3B). In contrast, when F9 EC cells were transfected after 3 days of RA-induced differentiation, the activity of the larger construct was fivefold higher than the activity of the smaller $m E l f 3-338 /+122$ construct (Fig. 3B). Together, these findings argue that at least one region outside the proximal promoter exerts a strong influence on Elf3 promoter activity in F9-differentiated cells.

\subsection{An upstream regulatory region elevates Elf3 promoter activity}

To identify the sequences responsible for elevated promoter activity, we examined the sequences between -2898 and -339 and between +122 and +703 for their contribution to Elf3 promoter activity. Initially, we created the promoter/reporter gene construct, $m E l f 3-338 /+703$ and compared its activity to that of $m E l f 3-338 /+122$. In F9differentiated cells, the activity of $m E l f 3-338 /+703$ was only slightly higher (approximately twofold) than that of $m$ Elf3 $-338 /+122$ (data not shown). Therefore, we focused our attention on the region between -2898 and -339 . Additional studies in the future will be needed to identify regulatory sequences located between +123 and +703 .
To identify sequences upstream of -338 that influence Elf3 promoter activity, progressively larger amounts of the Elf3 gene between -2898 and -2012 were deleted from 5' end of the promoter/reporter gene construct $m E l f 3-2898 /$ +703. Removal of the region between -2898 and -2347 did not reduce Elf3 promoter activity (Fig. 4A). However, deletion of the region upstream of -2012 reduced Elf 3 promoter activity (Fig. 4A). This suggested that the region of the Elf3 gene between -2347 and -2012 contained one or more $c i s$-regulatory elements that influence its promoter activity in F9-differentiated cells. This possibility was tested by deleting the region -2343 to -2013 to generate the construct $m E$ Elf $3-2898 /+703_{\Delta-2343 /-2013}$. When this construct was transfected into F9-differentiated cells, the luciferase activity generated was approximately fourfold lower than that observed with the parent, mElf3 $-2898 /+703$, construct (Fig. 4A).

The region between -2347 and -2012 was examined further using five additional promoter/reporter gene constructs, which incorporated portions of this region inserted into $m E l f 3-338 /+703$ upstream of the proximal promoter (Fig. 4B). Importantly, $m E l f 3-2236 /+703_{\Delta-2142 /-339}$, incorporating the $94 \mathrm{bp}$ sequence from -2236 to -2143 , exhibited nearly fourfold greater activity than $m E l f 3-338 /+703$. To verify the importance of the 94 bp region in the upregulation of the Elf 3 promoter when F9 EC cells undergo differentiation, we compared the activity of $m E l f 3-2236 /+703_{\Delta}-2142 /-339$ to that of $m E l f 3-338 /+703$ in F9 EC cells and F9-differentiated cells. As expected, there was no difference in the activity of these two constructs in F9 EC cells, whereas $m E$ lf $3-2236 /+703_{\Delta-2142 /-339}$ exhibited greater than sixfold higher activity in F9-differentiated cells (Fig. 4C). Hence, we focused the remainder of our efforts
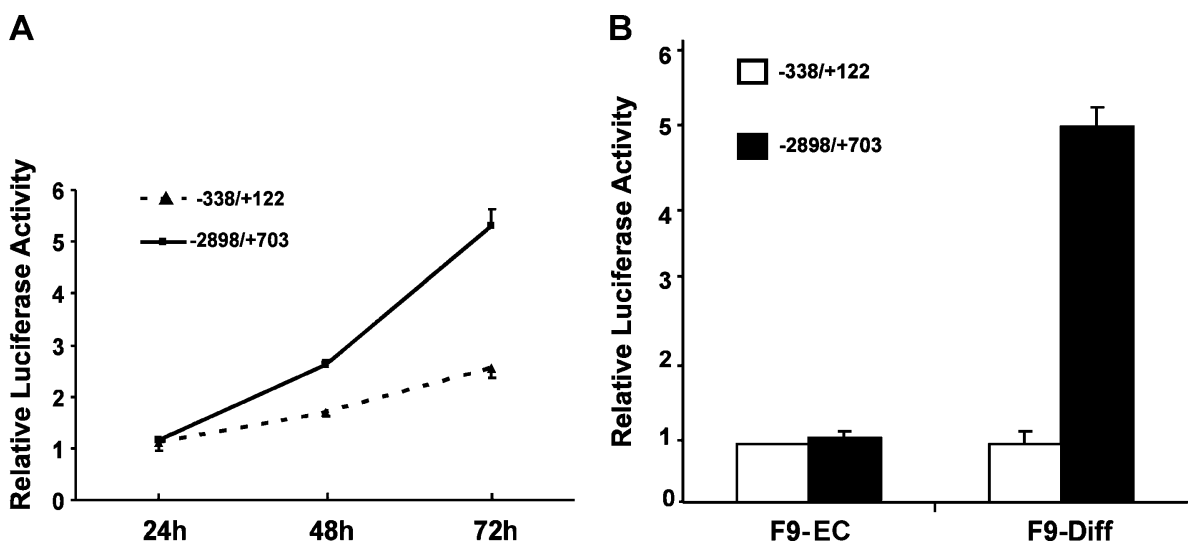

Fig. 3. An upstream distal enhancer significantly increases Elf3 promoter activity in F9-differentiated cells. F9 EC cells were transfected with either a short $-338 /+122$ or a long $-2898 /+703$ Elf3 promoter/reporter construct as described in Materials and methods. (A) F9 EC cells were transfected with the Elf3 promoter/reporter gene constructs shown. Five hours after transfection, half of the cells were induced to differentiate with $5 \mu \mathrm{M}$ RA. Luciferase assays were conducted 24, 48 or $72 \mathrm{~h}$ after transfection as described in Materials and methods. Results from duplicate F9 EC samples were averaged and used with individual differentiated cell samples to generate ratios of luciferase activity (F9-differentiated: F9 EC). The average \pm S.E.M. of these ratios is plotted. This experiment was repeated with triplicate samples with similar results. (B) F9 EC and F9-differentiated cells were transfected independently. Averages \pm S.E.M. are plotted for representative experiments, which were repeated multiple times with similar results. 
A

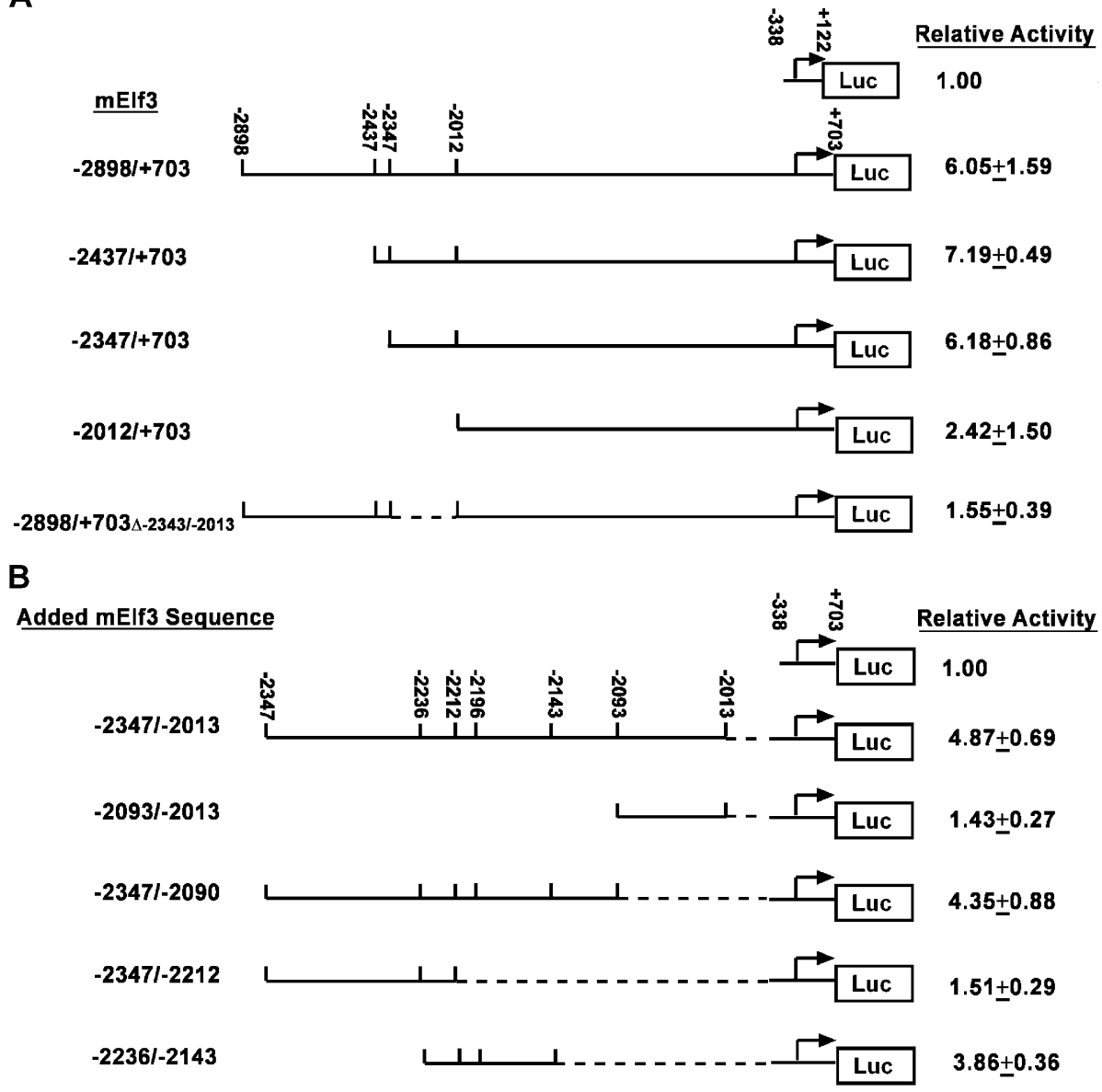

C

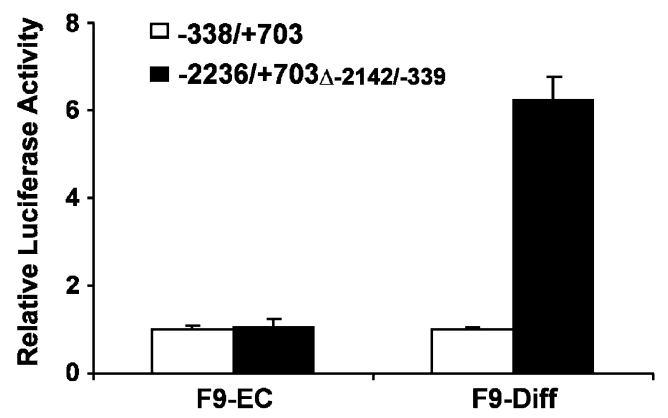

Fig. 4. Progressive deletion analysis of the Elf3 upstream flanking region. Promoter/reporter constructs containing the indicated regions of upstream flanking sequence were transfected into F9-differentiated cells and assayed for luciferase activity as described in Materials and methods. (A) Means from three similar experiments, each with triplicate samples expressed relative to $m E l f 3-338 /+122$, were used to generate averages \pm S.E.M. (B) Means from five similar experiments, each with triplicate samples expressed relative to $m E l f 3-338 /+703$, were used to generate averages \pm SEM. (C) F9 EC and F9-differentiated cells were transfected independently with either $m E l f 3-338 /+703$ or $m E l f 3-2236 /+703_{\Delta-2142 /-339}$ Elf3 promoter/reporter construct. For the purpose of comparison, the activity of the $m E l f 3-338 /+703$ was set to 1 . The values shown are averages \pm S.E.M. for triplicate samples from a representative experiment. This experiment was repeated and similar results were obtained.

in this study on the $94 \mathrm{bp}$ sequence between -2236 and 2143 .

\subsection{A 36 bp upstream region is required for elevated Elf3 promoter activity}

To identify specific DNA sequences within the sequence from -2236 to -2143 that are responsible for elevating
Elf3 promoter activity, saturation mutagenesis was employed. Specifically, one 3 bp mutant and fifteen 6 bp mutants were generated to sequentially disrupt the entire 94 bp sequence (Fig. 5A). For this purpose, the wild-type sequence for each of the fifteen $6 \mathrm{bp}$ mutants was replaced with the sequence $5^{\prime}$-CCCGGG- ${ }^{\prime}$. After transient transfection into F9-differentiated cells, the activity of each mutant construct was compared to the activity of $m E l f 3-2236 /$ 
A

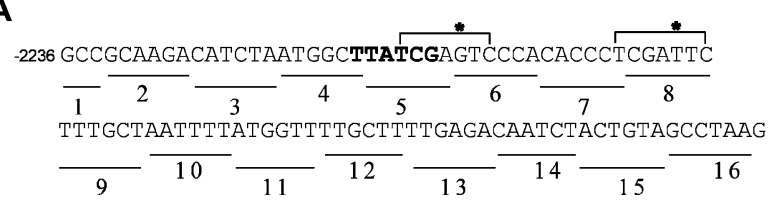

B

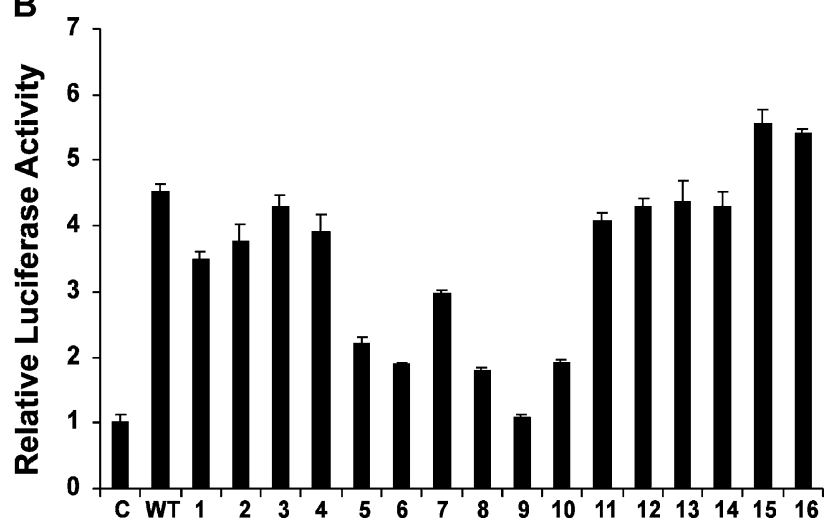

Fig. 5. Mutational analysis of the upstream 94 bp positive regulatory region. (A) The murine $94 \mathrm{bp}$ upstream enhancer region is shown. Sixteen sequences chosen for mutational analysis are sequentially numbered and underlined. A potential GATA site is shown in bold and a repeated sequence is marked with an upper bracket. The single difference in the $7 \mathrm{bp}$ repeat is labeled with an"*" (B) F9-differentiated cells were transfected with the wild-type $\left(m E l f 3-2236 /+703_{\Delta}-2142 /-339\right)$ or mutant promoter/reporter constructs containing Elf3 sequence from -2236 to -2143 inserted upstream of the $m E l f 3-338 /+703$ construct. In each numbered construct, the sequence to be mutated was replaced by the sequence 5'-CCCGGG-3' Cells were assayed for luciferase activity as described in Materials and methods. Values for the mutant constructs were compared to mElf3 - 338/ +703 , shown as "C", which was set to 1 . The values shown are averages \pm S.E.M. for triplicate samples from a representative experiment. This experiment was performed four times with similar results.

$+703_{\Delta}-2142 /-339$. This experiment identified a contiguous core of $36 \mathrm{bp}$ (mutants \#5 through \#10, - 2215 to -2180 ) that influenced Elf3 promoter activity (Fig. 5B). Each of the six mutant constructs, which encompass this 36 bp region, reduced promoter activity. The largest reduction was observed with mutant $\# 9$, which eliminated nearly all the activity of the $94 \mathrm{bp}$ sequence.

Thus far, efforts to identify the factors that bind to this 36 bp region have not been successful. Surprisingly, electrophoretic mobility shift assay (EMSA) failed to demonstrate specific binding to this region by any factors in nuclear extracts from F9-differentiated cells (data not shown). In silico analysis identified a region $(-2216$ to -2211$)$ within the $94 \mathrm{bp}$ sequence that differs by 1 bp from a consensus site for GATA factors (Fig. 5A). This was of interest, because GATA 4 and GATA 6 expression increases when EC cells undergo differentiation (Morrisey et al., 1996; Murakami et al., 1999). However, overexpression of neither GATA 4 nor GATA 6 influenced Elf3 promoter activity in F9-differentiated cells (data not shown). Interestingly, this region of the Elf3 gene contains near perfect $7 \mathrm{bp}$ repeats that differ from one another by only $1 \mathrm{bp}$, and which are separated from each other by 8 bp (Fig. 5A). However, these sequences do not appear to match the consensus sequence for any known eukaryotic transcription factor.

\section{Discussion}

Previous studies have shown that Elf3 mRNA increases at least sixfold after EC cells differentiate (Kim et al., 2002). However, changes in Elf3 protein levels in this model system have not been determined due to the lack of an antibody that is specific for mouse Elf3. In this study, we examined the DNA regulatory regions that influence the expression of the Elf3 gene. Specifically, we mapped the transcription start site of the murine Elf3 gene in F9differentiated cells and identified cis-regulatory elements located in the proximal promoter region as well as in a region $2 \mathrm{~kb}$ upstream of the transcription start site. The upstream regulatory region is localized to a $36 \mathrm{bp}$ sequence, which appears to play an important role in upregulation of Elf3 when F9 EC cells differentiate.

In this study, $5^{\prime}$-RACE identified a major transcription start site for the murine Elf3 gene in F9-differentiated cells at position +32 , which is $30 \mathrm{bp}$ downstream from the center of a TATA box at +2 . In our study, 16 of the 17 isolated clones identified a start site at position +32 , while the remaining clone corresponded to a start site at +33. In contrast to our findings, Tymms et al. (1997) used 5'-RACE and mapped the dominant start site in adult murine lung tissue to +51 . In their study, a total of seven clones were sequenced, and four of the seven clones identified a transcription start site at position +51 . The remaining three clones identified start sites at positions $+42,+44$ and +68 , respectively. An analysis of hundreds of genes by Bucher (1990) found the centerto-center spacing between a TATA box and the cap signal to be $30 \pm 2 \mathrm{bp}$. It is possible that multiple start sites are used for the Elf3 gene and that different start sites are used in different cell types. However, if functional, the TATA box would direct transcriptional initiation to the site that we identified in F9-differentiated cells rather than to the site found in adult lung. Two studies of the human ELF3 gene have mapped the transcription start site to -7 in adult liver cells (Oettgen et al., 1999) and -8 in a gastric cancer cell line (Park et al., 2001). Oettgen et al. (1999) point out that the difference in start site between the human and murine genes is likely due to the presence of a second TATA box centered at -39 in the human sequence, which is not present in the murine sequence.

The DNA sequences of the four promoter-proximal cis-regulatory elements (ESE, POU, $\kappa \mathrm{B}$ and CCAAT box) shown to be functional in F9-differentiated cells (Fig. 2) are $100 \%$ conserved between the mouse and human except for one base pair in the ESE site (Neve et al., 1998). This is also true for an overlapping CG box and USF site, but disruption of either site on its own or in 
combination failed to affect Elf3 promoter activity in F9differentiated cells (data not shown). In contrast to these sites, disruption of the $\kappa \mathrm{B}$ site and the ESE site reduced Elf3 promoter activity $43 \%$ and $48 \%$, respectively, (Fig. $2)$. Earlier studies demonstrated that both sites are functional in the case of the human gene. The $\mathrm{\kappa B}$ site was first shown to be functional in the case of the human ELF3 promoter using a mouse monocytic cell line (Rudders et al., 2001). This study also reported that the $\kappa \mathrm{B}$ site of the human ELF3 gene binds the p50 and p65 subunits of NF-kB in vitro (Rudders et al., 2001). Moreover, recent evidence argues strongly that p50 and p65 activate the human ELF3 promoter via the $\kappa \mathrm{B}$ site in vivo (Grall et al., 2003). Similar to these findings, we determined that $\mathrm{c}-\mathrm{Rel}$ and the p50 subunit of NF- $\mathrm{KB}$ present in nuclear extracts from F9-differentiated cells can bind to the $\mathrm{\kappa B}$ site in vitro (data not shown). The ESE site in the human ELF3 gene was also shown to be functional in an earlier study that used a human gastric tumor cell line (Park et al., 2001). Interestingly, disruption of the ESE site reduced the activity of the human ELF3 promoter approximately $80 \%$ in the gastric tumor cell line. Thus, the overall contribution of the ESE site may differ between the human and the murine gene and/or in different cell types $(48 \%$ for F9-differentiated cells vs. $80 \%$ for gastric tumor cells). Thus far, the nuclear factor shown previously to bind to the ESE site in vitro has not been identified (Park et al., 2001). Given that the ESE site influences Elf3 promoter activity in at least two cell types, the factor that binds to the ESE site warrants further study. However, it would appear that these studies would be best undertaken using gastric tumor cells.

In this study, we provide the first demonstration that the POU motif and the CCAAT box of this gene influence its promoter activity. Disruption of either site reduced Elf3 promoter activity more than $65 \%$ in F9-differentiated cells. In the case of the CCAAT box, we determined by chromatin immunoprecipitation that NF-Y binds to the promoter of the endogenous Elf3 gene in F9-differentiated cells, and we determined that NF-Y mediates the positive effect of the CCAAT box with the use of a dominantnegative mutant of NF-YA (Mantovani et al., 1994; data not shown). In other work not described in this report, we determined by electrophoretic mobility shift assay that the transcription factor Oct-1 in nuclear extracts prepared from F9-differentiated cells can bind to the POU motif of the Elf3 gene in vitro. Given the importance of the POU motif, future studies should determine whether Oct-1 binds to the POU motif in F9-differentiated cells and elevates Elf3 promoter activity in vivo.

The work reported in this study argues strongly that an upstream regulatory region plays an important role in elevating Elf3 promoter activity after EC cells undergo differentiation. Earlier studies demonstrated that the steady-state level of Elf3 mRNA increases after EC cells are induced to differentiate, reaching levels approximately sixfold higher after 3 days and eightfold higher after 5 days (Kim et al., 2002). In the present study, after 3 days of differentiation, the activity of promoter/reporter constructs containing the upstream region is elevated fivefold, while the activity of the proximal promoter appears to increase only twofold (Fig. 3A). We localized this positive regulatory region to a $94 \mathrm{bp}$ sequence between -2236 and -2143 by using a battery of Elf3 promoter/reporter gene constructs containing the proximal promoter and various portions of the $5^{\prime}$-flanking region of the Elf3 gene. In F9-differentiated cells, constructs containing this $94 \mathrm{bp}$ region have four- to sixfold higher activities than constructs without this region. This is in contrast to F9 EC cells, where both sets of constructs have similar activities (Fig. 4C). Together, our data argue that differentiation of EC cells significantly increases the activity of the Elf3 promoter and that full promoter activity is dependent on a distal regulatory region located $2 \mathrm{~kb}$ upstream of the transcription start site of Elf3. Interestingly, this regulatory region functions in a distance-independent manner, given that it functions even when it is placed $2 \mathrm{~kb}$ closer to the Elf3 promoter (Fig. 4B). We also believe that this region is likely to function in an orientation-independent manner, but this was not tested in this study.

Saturation mutagenesis of the $94 \mathrm{bp}$ regulatory region identified a core of $36 \mathrm{bp}$ from -2215 to -2180 , which is required for full Elf3 promoter activity. The importance of this $36 \mathrm{bp}$ region was demonstrated by six sequential 6 bp mutations, each of which reduced Elf3 promoter activity. Given that the size of this region is far greater than the size of a typical transcription factor binding site (4-10 bp), and the fact that cis-regulatory elements are often clustered in enhancers, we anticipate that more than one transcription factor binds to this regulatory region. Thus far, we have been unable to identify the factors involved. Efforts to identify proteins that bind to this 36 bp region using electrophoretic mobility shift assay have not been successful.

In conclusion, the work reported in this study establishes that the promoter of the murine Elf3 gene in F9-differentiated cells is controlled by at least four cis-regulatory elements located within 250 bp of the transcription start site. Moreover, we have identified a novel 36 bp regulatory region, which is located $2 \mathrm{~kb}$ upstream of the transcription start site. Importantly, this regulatory region exerts a strong influence on the Elf3 promoter activity in F9-differentiated cells and appears to play an important role in the upregulation of Elf3 gene expression after EC cells undergo differentiation. Given that these cells provide an excellent model for mammalian embryogenesis and that differentiation of EC cells strongly suppresses their tumorigenicity (Adamson and Graham, 1980), it will be of interest to better understand how the Elf3 gene is regulated in this model system. Such an understanding is likely to provide important insights into the regulation of this gene in both normal and diseased tissues. 


\section{Acknowledgements}

Craig Waters and Michelle Desler are thanked for technical assistance. This work was supported by a grant from the National Institutes of Health (CA 74771). Core facilities of the Eppley Institute were supported in part by a Cancer Center Support Grant (CA 36727).

\section{References}

Adamson, E.D., Graham, C.F., 1980. Loss of tumorigenicity and gain of differentiated function by embryonal carcinoma cell. Results Probl. Cell Differ. 11, 290-297.

Bucher, P., 1990. Weight matrix descriptions of four eukaryotic RNA polymerase II promoter elements derived from 502 unrelated promoter sequences. J. Mol. Biol. 212, 563-578.

Chang, C.H., Scott, G.K., Kuo, W.L., Xiong, X., Suzdaltseva, Y., Park, J.W., Sayre, P., Erny, K., Collins, C., Gray, J.W., Benz, C.C., 1997. ESX: a structurally unique Ets overexpressed early during human breast tumorigenesis. Oncogene 14, 1617-1622.

Chang, J., Lee, C., Hahm, K.B., Yi, Y., Choi, S.G., Kim, S.J., 2000. Overexpression of ERT(ESX/ESE-1/ELF3), an ets-related transcription factor, induces endogenous TGF-beta type II receptor expression and restores the TGF-beta signaling pathway in Hs578t human breast cancer cells. Oncogene 19, 151-154.

Choi, S.G., Yi, Y., Kim, Y.S., Kato, M., Chang, J., Chung, H.W., Hahm, K.B., Yang, H.K., Rhee, H.H., Bang, Y.J., Kim, S.J., 1998. A novel ets-related transcription factor, ERT/ESX/ESE-1, regulates expression of the transforming growth factor-beta type II receptor. J. Biol. Chem. $273,110-117$.

Dittmer, J., Nordheim, A., 1998. Ets transcription factors and human disease. Biochim. Biophys. Acta 1377, F1-F11.

Grall, F., Gu, X., Tan, L., Cho, J.Y., Inan, M.S., Pettit, A.R., Thamrongsak, U., Choy, B.K., Manning, C., Akbarali, Y., Zerbini, L., Rudders, S., Goldring, S.R., Gravallese, E.M., Oettgen, P., Goldring, M.B., Libermann, T.A., 2003. Responses to the proinflammatory cytokines interleukin-1 and tumor necrosis factor alpha in cells derived from rheumatoid synovium and other joint tissues involve nuclear factor kappaB-mediated induction of the Ets transcription factor ESE-1. Arthritis Rheum. 48, 1249-1260.

Isola, J.J., Kallioniemi, O.P., Chu, L.W., Fuqua, S.A., Hilsenbeck, S.G., Osborne, C.K., Waldman, F.M., 1995. Genetic aberrations detected by comparative genomic hybridization predict outcome in node-negative breast cancer. Am. J. Pathol. 147, 905-911.

Kim, J.H., Wilder, P.J., Hou, J., Nowling, T., Rizzino, A., 2002. Activation of the murine type II transforming growth factor-beta receptor gene: upregulation and function of the transcription factor Elf-3/Ert/Esx/Ese-1. J. Biol. Chem. 277, 17520-17530.

Kopp, J.L., Wilder, P.J., Desler, M., Kim, J.H., Hou, J., Nowling, T., Rizzino, A., 2004. Unique and selective effects of five Ets family members, Elf3, Ets1, Ets2, PEA3 and PU.1, on the promoter of the type II TGF-beta receptor gene. J. Biol. Chem. 279, 19407-19420.
Laudet, V., Hanni, C., Stehelin, D., Duterque-Coquillaud, M., 1999. Molecular phylogeny of the ETS gene family. Oncogene 18, 1351-1359.

Lee, H.J., Chang, J.H., Kim, Y.S., Kim, S.J., Yang, H.K., 2003. Effect of ets-related transcription factor (ERT) on transforming growth factor (TGF)-beta type II receptor gene expression in human cancer cell lines. J. Exp. Clin. Cancer Res. 22, 477-480.

Mantovani, R., Li, X.Y., Pessara, U., Hooft, v.H., Benoist, C., Mathis, D., 1994. Dominant negative analogs of NF-YA. J. Biol. Chem. 269, 20340-20346.

Morrisey, E.E., Ip, H.S., Lu, M.M., Parmacek, M.S., 1996. GATA-6: a zinc finger transcription factor that is expressed in multiple cell lineages derived from lateral mesoderm. Dev. Biol. 177, 309-322.

Murakami, A., Thurlow, J., Dickson, C., 1999. Retinoic acid-regulated expression of fibroblast growth factor 3 requires the interaction between a novel transcription factor and GATA-4. J. Biol. Chem. 274, $17242-17248$.

Neve, R., Chang, C.H., Scott, G.K., Wong, A., Friis, R.R., Hynes, N.E., Benz, C.C., 1998. The epithelium-specific ets transcription factor ESX is associated with mammary gland development and involution. FASEB J. $12,1541-1550$.

Ng, A.Y., Waring, P., Ristevski, S., Wang, C., Wilson, T., Pritchard, M., Hertzog, P., Kola, I., 2002. Inactivation of the transcription factor Elf3 in mice results in dysmorphogenesis and altered differentiation of intestinal epithelium. Gastroenterology 122, 1455-1466.

Nowling, T., Bernadt, C., Johnson, L., Desler, M., Rizzino, A., 2003. The coactivator $\mathrm{p} 300$ associates physically with and can mediate the action of the distal enhancer of the FGF-4 gene. J. Biol. Chem. 278, 13696-13705.

Oettgen, P., Alani, R.M., Barcinski, M.A., Brown, L., Akbarali, Y., Boltax, J., Kunsch, C., Munger, K., Libermann, T.A., 1997. Isolation and characterization of a novel epithelium-specific transcription factor, ESE-1, a member of the ets family. Mol. Cell. Biol. 17, 4419-4433.

Oettgen, P., Barcinski, M., Boltax, J., Stolt, P., Akbarali, Y., Libermann, T.A., 1999. Genomic organization of the human ELF3 (ESE-1/ESX) gene, a member of the Ets transcription factor family, and identification of a functional promoter. Genomics 55, 358-362.

Park, S.H., Kim, Y.S., Park, B.K., Hougaard, S., Kim, S.J., 2001. Sequence-specific enhancer binding protein is responsible for the differential expression of ERT/ESX/ELF-3/ESE-1/jen gene in human gastric cancer cell lines: implication for the loss of TGF-beta type II receptor expression. Oncogene 20, 1235-1245.

Reddy, S.P., Vuong, H., Adiseshaiah, P., 2003. Interplay between proximal and distal promoter elements is required for squamous differentiation marker induction in the bronchial epithelium: role for ESE-1, Sp1, and AP-1 proteins. J. Biol. Chem. 278, 21378-21387.

Rudders, S., Gaspar, J., Madore, R., Voland, C., Grall, F., Patel, A., Pellacani, A., Perrella, M.A., Libermann, T.A., Oettgen, P., 2001. ESE-1 is a novel transcriptional mediator of inflammation that interacts with NFkappa B to regulate the inducible nitric-oxide synthase gene. J. Biol. Chem. 276, 3302-3309.

Tymms, M.J., Ng, A.Y., Thomas, R.S., Schutte, B.C., Zhou, J., Eyre, H.J., Sutherland, G.R., Seth, A., Rosenberg, M., Papas, T., Debouck, C., Kola, I., 1997. A novel epithelial-expressed ETS gene, ELF3: human and murine cDNA sequences, murine genomic organization, human mapping to $1 \mathrm{q} 32.2$ and expression in tissues and cancer. Oncogene $15,2449-2462$. 